\title{
Serine/Threonine-Protein Kinase LATS1
}

National Cancer Institute

\section{Source}

National Cancer Institute. Serine/Threonine-Protein Kinase LATS1. NCI Thesaurus. Code C26344.

Serine/threonine-protein kinase LATS1 (1130 aa, 127 kDa) is encoded by the human LATS1 gene. This protein plays a role in both mitosis and serine/threonine phosphorylation. 